\title{
Sub-THz nonresonant detection in AIGaN/GaN heterojunction FETs
}

\author{
A.G. Golenkov ${ }^{1}$, K.S. Zhuravlev ${ }^{2}$, J.V. Gumenjuk-Sichevska ${ }^{1}$, I.O. Lysiuk ${ }^{1}$, F.F. Sizov ${ }^{1}$ \\ ${ }^{I}$ V. Lashkaryov Institute of Semiconductor Physics, NAS of Ukraine, \\ 41, prospect Nauky, 03028 Kyiv, Ukraine \\ E-mail: golenkov.o@gmail.com; gumenjuk@gmail.com; Lysiuk@gmail.com; sizov@isp.kiev.ua \\ ${ }^{2}$ A. Rzhanov Institute of Semiconductor Physics, Siberian Branch of RAS, \\ 13, pr. Lavrentieva, Novosibirsk, 630090 Russia; e-mail: zhur@thermo.isp.nsc.ru
}

\begin{abstract}
Un-cooled AlGaN/GaN-based heterojunction field-effect transistors (HFET) designed on sapphire (0001) substrates were considered as $140 \mathrm{GHz}$ direct detection detectors without any specially attached antennas. The noise equivalent power $(N E P)$ of these detectors was $\sim 10^{-10} \mathrm{~W} / \mathrm{Hz}^{1 / 2}$ in the observed radiation frequency range at ambient temperatures. It has been shown that the ultimate value for the AlGaN/GaN HFET detectors (in $0.25-\mu \mathrm{m}$ technology) can reach $N E P_{\text {opt }} \approx 6 \cdot 10^{-12} \mathrm{~W} / \mathrm{Hz}^{1 / 2}$, and it is 3 -fold lower than that for Si MOSFET (in 0.35- $\mu \mathrm{m}$ technology).
\end{abstract}

Keywords: sub-THz detectors, heterojunction FET, AlGaN/GaN heterostructure, Si MOSFET, sensitivity.

Manuscript received 06.10.14; revised version received 10.12.14; accepted for publication 19.02.15; published online 26.02.15.

\section{Introduction}

Terahertz (THz) technologies for radiation frequencies loosely defined from $v \sim 0.1$ to $10 \mathrm{THz}[1,2](\lambda \sim$ $3 \mathrm{~mm} . .30 \mu \mathrm{m})$ are now the emerging ones, which promises wide choice for potential applications in vision systems, high speed wireless communications, spectroscopy, medicine, security, pharmacology, etc. [3, 4]. They can give relatively high resolution images. At the same time, the $\mathrm{THz}$ radiation is innocuous as it is not ionizing, e.g. for the human body in the case of active imaging. Along with sources, important components of these technologies are detectors. Uncooled detectors implemented as an integrated array are required to obtain real time imaging in many applications.

AlGaN/GaN-based heterojunction field-effect transistors (HFETs) have been reported as one of the most promising devices for high-power and highfrequency applications due to material properties of nitride-based compounds such as wide band-gap, high breakdown voltage and high two-dimensional electron gas (2DEG) density (see e.g. [5-7]). In recent years, attention to AlGaN/GaN-based HFETs is attracted because of their high potential applicability as sub$\mathrm{THz} / \mathrm{THz}$ detectors [8-10].

\section{Advantages of AlGaN/GaN HFETs}

The advantages of AlGaN/GaN HFETs over Si MOSFETs are higher channel electron mobility $\mu_{n}$ (by the factor approximately 4) and lower gate material resistivity (by the factor close to 100). Both of these factors provide the lower detector resistance $R_{0}$ and, as a result, the lower thermal (Johnson) noise $U_{\mathrm{J}}$, which is the 
principal noise of these detectors at biases around zero. It can be explained using the equation $R_{0}=L /\left(W \cdot \mu_{n} \cdot C_{O X}^{\prime} \cdot n \cdot \varphi_{t}\right) \quad[11]$ for $\mathrm{Si}$ MOSFETs where the resistance $R_{0}$ is in direct proportion to the channel length $L$, inversely to the channel width $W$ and mobility $\mu_{n}$. Parameters $C_{O X}^{\prime}, n, \varphi_{t}$ are the oxide capacity, the $I-V$ characteristic slope in the sub-threshold region, the thermal potential, respectively. Usually, the length $L$ is designed as small as manufacturing design rules allow. But the width $W$ can be optimized. To decrease resistance $R_{0}$, the width $W$ should be increased. At the same time, the width $W$ can not be very wide, because the gate parasitic serial resistance $R_{S}$ becomes large according to [7]:

$R_{S}=r_{0}+r_{1} / W+r_{2} \cdot W /(3 L)$,

where $r_{0}$ is the resistance of the contacts between metal and gate layers $(\sim 5 \Omega), r_{1}$ - transistor source resistivity, and $r_{2}$ - gate material resistivity. Due to the fact that AlGaN/GaN HFET gate has a Schottky barrier, its metallic gate resistivity $r_{2}$ is considerably smaller than the polysilicon gate resistivity of $\mathrm{Si}$ MOSFET (see Fig. 1a). Typically, the Si MOSFET $r_{1}$ and $r_{2}$ values are $r_{1} \sim 400 \Omega \cdot \mu \mathrm{m}, r_{2} \sim 40 \Omega$ (according to the $0.35-\mu \mathrm{m}$ technology design rules), and the AlGaN/GaN HFET value $r_{2}<0.1 \Omega$. To avoid power losses, the value of $R_{S}$ have to be considerably lower than the antenna radiation resistance $R_{A R}(\sim 100 \Omega)$.

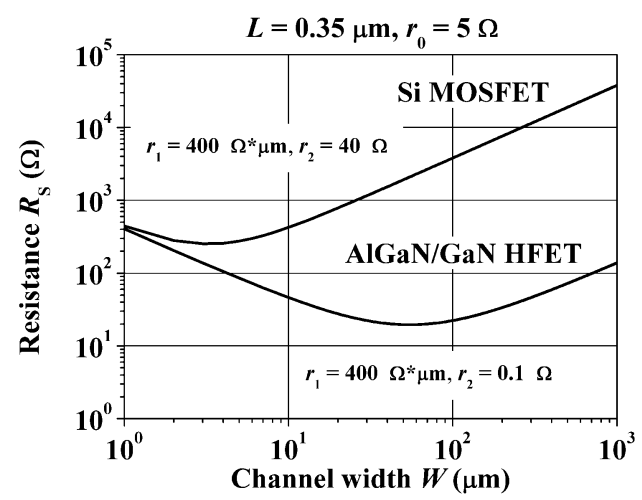

a

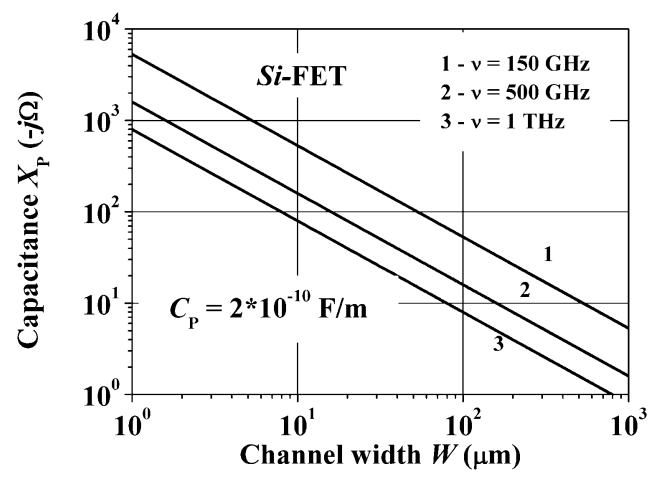

b

Fig. 1. Modeled channel width dependences of the parasitic serial resistance $(a)$ and the shunting capacitance $(b)$.
The width $W$ limited also by parasitic shunting capacitance $X_{P}=-j /\left(2 \pi v \cdot W \cdot C_{P}^{\prime}\right)$ appeared between the transistor gate and the source [12] ( $v$ is irradiation frequency), where $C_{P}^{\prime}$ is the shunting capacity density on the width unit (Fig. 1b). Value $C_{P}^{\prime}$ depends on the FET production technology (typically, the value $C_{P}^{\prime} \approx$ $2 \cdot 10^{-10} \mathrm{~F} / \mathrm{m}$ for $0.35-\mu \mathrm{m}$ design rules).

It seems that the influence of the channel width $W$ on capacitance $X_{P}$ for AlGaN/GaN HFET and $\mathrm{Si}$ MOSFETs is the same.

\section{Optical NEP and sensitivity of the rectifying type detectors}

Sub-THz detectors based on zero-biased Schottky-barrier diodes (SBD) and field effect transistors (FET), heterojunction field effect transistors (HFET), high electron mobility transistors (HEMT) in low frequency (nonresonant) long gate regime [13] are detectors of rectifying type and can be described in a similar way [12].

Most of the previous publications (e.g. $[14,15])$ concentrate their attention on the electrical noise equivalent power $(N E P)$ rather than optical $N E P_{\text {opt }}$. The latter one takes into account antenna properties and its matching efficiency with detector. The optical $N E P_{\text {opt }}$ and sensitivity $\Re_{\mathrm{V}, \text { opt }}$ of the rectifying type detectors can be found from the modified equations adduced [12], according to which one can obtain the relatively simple analytical expressions for $N E P_{\mathrm{opt}}$ and sensitivity $\mathfrak{R}_{\mathrm{V}, \text { opt }}$ are

$$
\begin{aligned}
& N E P_{\mathrm{opt}}=\frac{4 \cdot n \cdot q^{1 / 2} \cdot \varphi_{t}^{3 / 2} \cdot R_{0}^{1 / 2}}{R_{A, R}} \cdot \frac{f_{\sigma}^{1 / 2}}{F} \cdot \frac{1}{\xi_{Z} \cdot \xi_{\mathrm{opt}} \cdot e_{c d} \cdot D_{0}}, \\
& \mathfrak{R}_{V, \mathrm{opt}}=\frac{R_{A R}}{2 \cdot n \cdot \varphi_{t}} \cdot \frac{F}{f_{\sigma}} \cdot \eta_{L} \cdot \xi_{Z} \cdot \xi_{\mathrm{opt}} \cdot e_{c d} \cdot D_{0},
\end{aligned}
$$

where $n$ is the $I-V$ characteristic slope in the sub-threshold region (FET, HFET, HEMT) or ideality factor for SBD, $q$, $R_{0}, e_{c d}, D_{0}, \varphi_{t}=k_{\mathrm{B}} T / q, k_{\mathrm{B}}, T$ are the elementary electronic charge, detector resistance, antenna radiation efficiency, antenna directivity, thermal potential, Boltzmann constant, and temperature, respectively. The coefficient $\eta_{L}=\left|Z_{L} /\left(R_{D}+Z_{L}\right)\right|$ defines the voltage divider between detector resistance $R_{D}$ and external load impedances $Z_{\mathrm{L}}$; the coefficient $\xi_{\mathrm{Z}}$ describes sub-THz frequency voltage divider between antenna $Z_{A}$ and detector $Z_{\text {int }}$ wave impedances in compliance with equation $\xi_{Z}=\left|\frac{X_{P} \| Z_{\text {int }}}{Z_{A}+R_{S}+X_{P} \| Z_{\text {int }}}\right|^{2}$, the coefficient $\xi_{\text {opt }}$ is equal to $\xi_{\text {opt }}=\lambda^{2} /\left(4 \pi \cdot A_{\text {opt }}\right)$ ( $\lambda$ is the irradiation wavelength, $A_{\text {opt }}$ is the detector physical area). 
For FET, HFET, and HEMT, the function $f_{\sigma}$ characterizes dependence of the conductivity $\sigma$ on the gate-source voltage $V_{G S}$ is

$$
f_{\sigma}(x)=2 \cdot \exp (x / 2) \cdot \ln [1+\exp (x / 2)] /[1+\exp (x / 2)],
$$

where $x=\left(V_{G S}-V_{\text {th }}\right) /\left(n \cdot \varphi_{t}\right), \quad V_{\text {th }}$ is the threshold voltages of the transistor. The function $F$ is $F(x)=$ $(2-n) \cdot\left(d f_{\sigma} / d x\right)$, and the formula for internal impedance $Z_{\text {int }}$ is $Z_{\text {int }}=(1-j) \sqrt{R_{0} /\left(4 \pi v \cdot W \cdot L \cdot C_{O X}^{\prime}\right)} / f_{\sigma}^{1 / 2}[12]$.

For zero-biased SBD detectors $f_{\sigma}(0)=F(0)=1$.

The minimum value of the function $f_{\sigma}^{1 / 2} \cdot\left(d f_{\sigma} / d x\right)^{-1} \approx 1.75$ and the maximum value of the function $\left(1 / f_{\sigma}\right) \cdot\left(d f_{\sigma} / d x\right)=1$ (Fig. 2) define optimum $N E P_{\text {opt }}$ and sensitivity $\Re_{V, \text { opt }}$.

Using the expressions (1), (2) and assumptions $\eta_{L}=\xi_{Z}=\xi_{\text {opt }}=D_{0}=e_{c d}=1$, one can estimate the ultimate performance values $N E P_{\text {opt }}$ and sensitivity $\Re_{V \text {,opt }}$ of rectifying type detectors as

$$
\begin{aligned}
& \mathfrak{R}_{V, \text { opt }} \approx 1500 \frac{\mathrm{V}}{\mathrm{W}} \cdot\left(\frac{1.3}{n}\right) \cdot\left(\frac{R_{A R}}{100}\right) \cdot\left(\frac{300}{T}\right) \cdot \frac{F}{f_{\sigma}}, \\
& N E P_{\mathrm{opt}} \approx 2.6 \cdot 10^{-12} \frac{\mathrm{W}}{\mathrm{Hz}^{1 / 2}} \cdot \frac{n}{1.3} \cdot \frac{100}{R_{A R}} \times \\
& \times\left(\frac{T}{300}\right)^{3 / 2} \cdot\left(\frac{R_{0}}{1000}\right)^{1 / 2} \cdot \frac{f_{\sigma}^{1 / 2}}{F} .
\end{aligned}
$$

Taking into account the optimum values of the functions $f_{\sigma}^{1 / 2} / F$ and $F / f_{\sigma}$ are $\sim 1.75 /(2-n)$ and $2-n$, respectively, from (3) and (4) at $n=1.3, R_{A R}=$ $100 \Omega, R_{0}=1000 \Omega$ (for acceptable channel width in the $0.25-\mu \mathrm{m}$ technology), and $T=300 \mathrm{~K}$, we estimate the ultimate optical of the $\mathrm{AlGaN} / \mathrm{GaN}$ HFET as $N E P_{\text {opt }} \approx$ $6.5 \cdot 10^{-12} \mathrm{~W} / \mathrm{Hz}^{1 / 2}$ and sensitivity as $\Re_{V, \mathrm{opt}} \approx 1000 \mathrm{~V} / \mathrm{W}$.

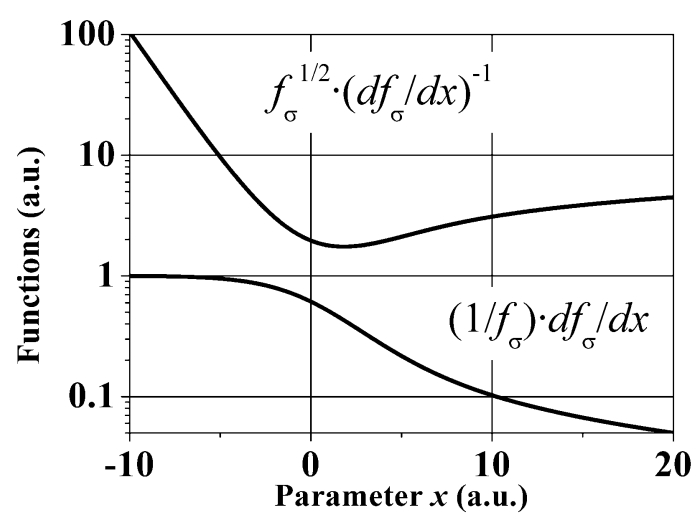

Fig. 2. Functions to calculate $N E P_{\text {opt }}$ and sensitivity $\Re_{V, \text { opt }}$.
In comparison with $\mathrm{AlGaN} / \mathrm{GaN}$ HFET, the ultimate optical $N E P_{\text {opt }}$ of Si MOSFETs will be worse by the factor $\sim 3$ because its minimal resistance $R_{0} \sim 7.4 \mathrm{k} \Omega$ (for an acceptable channel width in the $0.35-\mu \mathrm{m}$ technology): for $\mathrm{Si}$ MOSFETs is equal to $N E P_{\mathrm{opt}} \approx$ $1.8 \cdot 10^{-11} \mathrm{~W} / \mathrm{Hz}^{1 / 2}$.

\section{Samples}

SBD and silicon field effect transistors are rather widely tested as sub-THz/THz uncooled detectors [2]. Here, to test heterostructure field effect transistors as sub$\mathrm{THz} / \mathrm{THz}$ detectors, commercial AlGaN/GaN HFETs were chosen.

The $\mathrm{AlGaN} / \mathrm{GaN}$ heterostructures were grown on sapphire (0001) substrate of $d=400 \mu \mathrm{m}$ thickness by molecular beam epitaxy method (MBE) using CBE RIBER 32 equipment at the temperature $T \sim 900{ }^{\circ} \mathrm{C}$. The cross section of the heterostructure is shown schematically in Fig. 3. The room temperature sheet electron concentration in the two-dimensional (2D) channel layer is $n_{300 \mathrm{~K}} \approx 1.2 \cdot 10^{13} \mathrm{~cm}^{-2}$, and the mobility is $\mu_{300 \mathrm{~K}} \approx 1.46 \cdot 10^{3} \mathrm{~cm}^{2} / \mathrm{V} \cdot \mathrm{s}$. They were measured using the Van der Pauw method in the magnetic field $H=0.5 \mathrm{~T}$.

To decrease the substrate influence of detector response on radiation frequency and antenna gain, the $\mathrm{Al}_{2} \mathrm{O}_{3}$ substrates were polished to the thickness $d=$ $175 \mu \mathrm{m}$. Here, the contact wires to HFETs pads serve as antennas. At this substrate thickness, the optical thickness $d \times(\varepsilon \approx 9.3)^{1 / 2} \approx 0.53 \mathrm{~mm}$ ( $\varepsilon$ is the dielectric permittivity), which is less than the radiation wavelength in air $\lambda=2.14 \mathrm{~mm}$, and though it does not satisfy the inequality $d(\varepsilon \approx 9.3)^{1 / 2}<0.1 \mathrm{~mm}$ to suppress the resonances in the substrate still it is making the response not strongly dependent on the radiation frequency

As for sub-THz detectors, sapphire substrate is not very appropriate, as its dielectric permittivity is high (perpendicular to $\mathrm{c}$ axis $\varepsilon_{\perp} \approx 9.3$, and parallel to $c$ axes $\varepsilon_{\| \mid} \approx 11.5$ ), which leads to the large optical thickness and appearance of radiation modes within the substrate making the response dependent on the radiation frequency [16]. To exclude the influence of the substrate on frequency dependence of the registered signal, the estimations have shown the thickness of sapphire substrate $d$ should not exceed $d=0.25 \lambda / \varepsilon^{1 / 2} \approx$ $150-175 \mu \mathrm{m}$ for the radiation frequency $140 \mathrm{GHz}$ and, for example, twice less for $300 \mathrm{GHz}$. To decrease the substrate influence of detector response on radiation frequency, the $\mathrm{Al}_{2} \mathrm{O}_{3}$ substrates were back thinned to the thickness $d=175 \mu \mathrm{m}$. Besides the suppression of the resonance modes in the substrate, and improving the antenna gain, it makes the system less sensitive to changing the frequency of radiation [17].

Shown in Fig. 4 is the linear array of five separate AlGaN/GaN HFETs detectors. Here, the contact wires to HFETs contact pads serve as antennas. We measured the 
photo-responses $V_{\text {sig }}$ of these detectors on the frequency $v=140 \mathrm{GHz}$ at the ambient temperature. The sensitivities $\mathfrak{R}_{V}[\mathrm{~V} / \mathrm{W}]$ were calculated.

At the threshold gate-source value $V_{t h} \approx-4.3 \mathrm{~V}$ of the studied transistors the channel resistance $R_{c h} \approx 200 \Omega$ (ratio $L / W \approx 2.5 \cdot 10^{-3}$, width $W=100 \mu \mathrm{m}$ ).

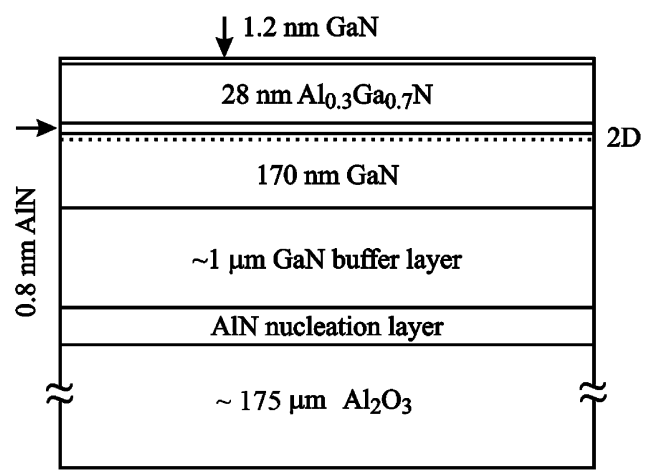

Fig. 3. The schematic cross-section of the $\mathrm{AlGaN} / \mathrm{GaN}$ heterostructure.

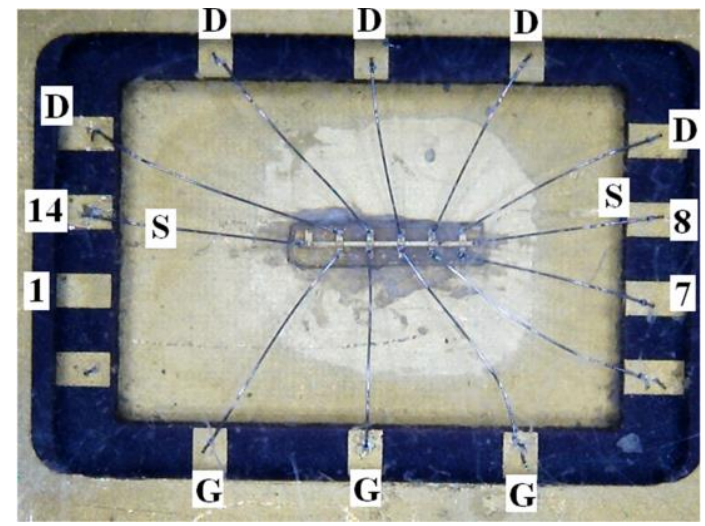

Fig. 4. The general view of five elements AlGaN/GaN HFET.

\section{Results of the measurements}

The noise equivalent power $N E P$ is one of the most important parameters that characterizes detector. It determined as $N E P=V_{\text {noise }} / \Re_{V}\left(\mathrm{~W} / \mathrm{Hz}^{1 / 2}\right)$, where $V_{\text {noise }}$ is the detector noise voltage, and the voltage sensitivity $\Re_{V}=V_{\mathrm{sig}} / P_{\mathrm{THz}}, P_{\mathrm{THz}}$ is the radiation power falling down onto detector.

To estimate the detector sensitivity, the relative power distribution from the $140 \mathrm{GHz}$ source was mapped using the sub- $\mathrm{THz} / \mathrm{THz}$ detector at sample displacement. Experimental points satisfy well the Fraunhofer diffraction intensity dependence with the Airy disk diameter $\approx 2.44 \cdot \lambda_{0} \cdot F / \# \approx 13 \mathrm{~mm}$ (for optical system $f$-number $F / \# \approx 2.5)$. Concentrated in the Airy disk is approximately $84 \%$ of radiation power from the source, reflection and absorption losses of optical system were estimated as $70 \%$, and the absolute power from the source was $P \approx 15 \mathrm{~mW}$. The power at the detector in its centre was calculated according to the method presented in [18] using the Gauss function that fits well the power distribution. The radiation power density $I_{\mathrm{THz}} \approx$ $0.071 \mathrm{~W} / \mathrm{cm}^{2}$ at the sample displacement according to these calculations. Note that the maximum possible antenna effective area $A_{\text {eff }}=G \cdot \lambda^{2} /(4 \pi) \approx 0.36 \mathrm{~mm}^{2}$ [19] (here, the antenna gain $G$ was taken as $G=1$ ).

For FET THz detectors, the main noise $V_{\text {noise }}$ in the zero-bias regime is the thermal one [14]. It was calculated from the equation $V_{\text {noise }}=\sqrt{4 \cdot k_{\mathrm{B}} \cdot T \cdot R_{c h}}$ where dependence of the channel resistance $R_{c h}$ on the gatesource voltage $V_{G S}$ was measured. These values were used for NEP estimations. The calculated thermal noise is shown in Fig. 5. Due to the intrinsic noise of the lockin amplifier (Stanford SR 830) used to detect, the signal is not less than $6 \mathrm{nV} / \mathrm{Hz}^{1 / 2}$, the lowest detector noise cannot be measured. The minimal $N E P \approx 10^{-10} \mathrm{~W} / \mathrm{Hz}^{1 / 2}$ is observed at the gate-source voltage $V_{G S}=-4.6 \mathrm{~V}$ (Fig. 5)

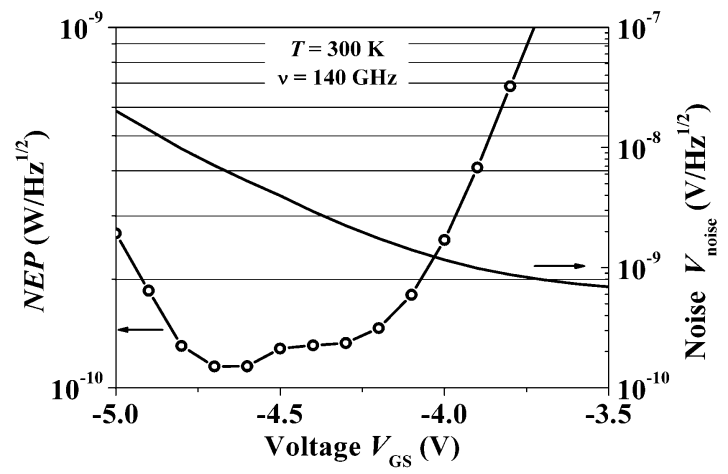

Fig. 5. The $N E P$ and calculated thermal noise dependences on the gate-source voltage for AlGaN/GaN HFET.

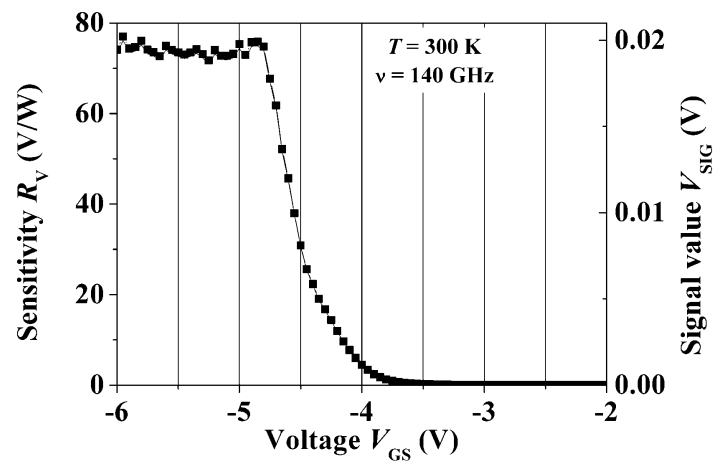

Fig. 6. The signal $V_{\text {sig }}$ and corresponded sensitivity $\mathfrak{R}_{V}$ dependences on the gate-source voltage for AlGaN/GaN HFET. 


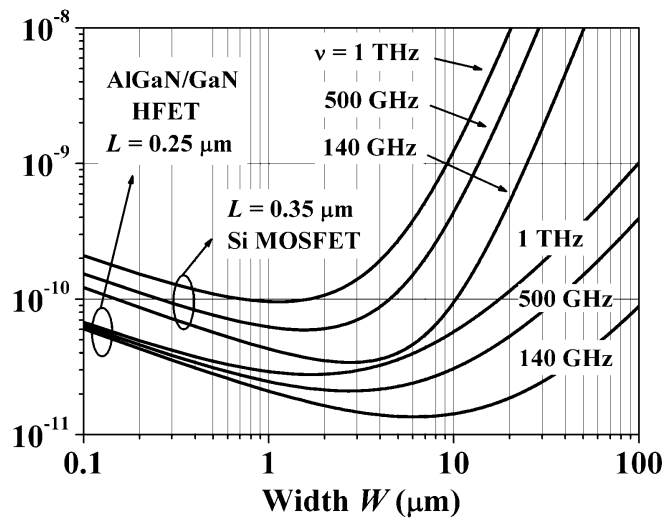

Fig. 7. Calculated using the equation (2) NEP dependences on the width $W$ of the transistors for AlGaN/GaN HFET $(0.25-\mu \mathrm{m}$ design rules) and Si MOSFET $(0.35-\mu \mathrm{m}$ design rules) at various irradiation frequencies.

The signal $V_{\text {sig }}$ dependence for one of HFET on the gate-source voltage is shown in Fig. 6. One can see saturation of this dependence [20] at high values of $V_{G S}$ (left part of Fig. 6). It is conditioned with the equations for signal $V_{s i g} \sim\left(\sigma_{c h}\right)^{-1} \cdot\left(d \sigma_{c h} / d V_{G S}\right)$ and the channel conductivity $\sigma_{c h}=\sigma_{0} \cdot \exp \left[\left(V_{G S}-V_{t h}\right) /\left(n k_{\mathrm{B}} T\right)\right]$ in the sub-threshold region, $\sigma_{0}$ is the channel conductivity at threshold value. The sensitivity $\mathfrak{R}_{V}$ dependence on $V_{\mathrm{GS}}$ used for NEP estimations is shown in Fig. 6. These values of sensitivity $\mathfrak{R}_{V}$ together with relatively low noise level of AlGaN/GaN HFET can provide the values of NEP which are comparable with those for other uncooled detectors [2].

\section{Discussion}

The experimentally measured value $N E P \approx$ $10^{-10} \mathrm{~W} / \mathrm{Hz}^{1 / 2}$ is less than its ultimate value by the factor of 10 , and it agrees with the results of calculations presented in Fig. 7. The optimal channel width $W_{0}$ at the irradiation frequency $140 \mathrm{GHz}$ is $6 \mu \mathrm{m} \quad(N E P \approx$ $\left.1.35 \cdot 10^{-11} \mathrm{~W} / \mathrm{Hz}^{1 / 2}\right)$. But the width of investigated (commercial) samples was $100 \mu \mathrm{m}$ (very high shunting capacitance). Mathematically, it is defined by the coefficient $\xi_{Z}: \xi_{Z}=0.54(W=6 \mu \mathrm{m})$, and $\xi_{Z}=0.02(W=$ $100 \mu \mathrm{m})$.

To calculate the dependences (Fig. 7), we used the following parameters for AlGaN/GaN HFET $L=$ $0.25 \mu \mathrm{m}, C_{O X}^{\prime} \approx 2.73 \cdot 10^{-3} \mathrm{~F} / \mathrm{m}^{2}, C_{P}^{\prime} \approx 1.23 \cdot 10^{-10} \mathrm{~F} / \mathrm{m}$, $n=1.3, x=3, \mu_{n}=1500 \mathrm{~cm}^{2} / \mathrm{V} \cdot \mathrm{s}, Z_{A}=100-100 j, r_{0}=$ $5 \Omega, r_{1}=400 \Omega \cdot \mu \mathrm{m}, r_{2}=0.1 \Omega, \xi_{\mathrm{opt}}=e_{c d}=D_{0}=1$, and for Si MOSFET $L=0.35 \mu \mathrm{m}, C_{O X}^{\prime} \approx 4.5 \cdot 10^{-3} \mathrm{~F} / \mathrm{m}^{2}$, $C_{P}^{\prime}=2 \cdot 10^{-10} \mathrm{~F} / \mathrm{m}, n=5, x=3, \mu_{n}=400 \mathrm{~cm}^{2} / \mathrm{V} \cdot \mathrm{s}, Z_{A}=$ $100-100 j, \quad r_{0}=5 \Omega, \quad r_{1}=400 \Omega \cdot \mu \mathrm{m}, \quad r_{2}=40 \Omega$, $\xi_{\mathrm{opt}}=e_{\mathrm{cd}}=D_{0}=1$.
The values of $N E P$ presented in [21] for InGaAs FETs $\quad\left(N E P \sim 8 \cdot 10^{-12} \mathrm{~W} / \mathrm{Hz}^{1 / 2}\right) \quad$ seems as overestimated ones as for $V_{\text {sig }}$ and $N E P$ calculations the effective antenna area was taken a physical area of the structure with the chain of four FETs only (without area of the contacts), which is several times lower than the effective area of the antenna $\lambda^{2} / 4 \pi[16,19]$ that was taken in this article as a normalization constant.

\section{Conclusions}

Nonresonant detection of the sub- $\mathrm{THz}$ radiation in AlGaN/GaN HFETs has been investigated. It has been shown the main its advantages in comparison with $\mathrm{Si}$ MOSFETs are higher channel electron mobility and lower gate material resistivity (metallic one in contrast to polysilicon in Si MOSFETs). As a result, the resistance, thermal noise, and noise equivalent power of the $\mathrm{AlGaN} / \mathrm{GaN}$ HFET detectors are less than their values in Si MOSFETs one by factors of $\sim 10, \sim 3$, and $\sim 3$, respectively.

Equations for optical voltage sensitivity $\mathfrak{R}_{V, \text { opt }}$ and optical $N E P_{\text {opt }}$ allow calculation of their ultimate values for the AlGaN/GaN HFET detectors, which are equal to $\Re_{V, \text { opt }} \approx 1000 \mathrm{~V} / \mathrm{W}$ and $N E P_{\mathrm{opt}} \approx 6.5 \cdot 10^{-12} \mathrm{~W} / \mathrm{Hz}^{1 / 2}$, respectively, at room temperatures for $0.25-\mu \mathrm{m}$ technology design rules.

The values of sensitivity $\mathfrak{R}_{V, \mathrm{opt}} \sim 80 \mathrm{~V} / \mathrm{W}$ and noise equivalent power $N E P_{\text {opt }} \sim 10^{-10} \mathrm{~W} / \mathrm{Hz}^{1 / 2}$ obtained in the samples investigated are sufficiently worse as compared to their estimated ultimate values and can be explained by lack of the special designed receiving antennas to the detectors and large transistor width though these values are typical for other uncooled THz/sub-THz detectors.

The optimal width of the transistors depends on irradiation frequency and technology design rules. For AlGaN/GaN HFET detectors manufactured using 0.25$\mu \mathrm{m}$ technology, the optimal width $W_{0}$ is equal to $6,2.7$, and $1.6 \mu \mathrm{m}$ for frequencies $140 \mathrm{GHz}, 500 \mathrm{GHz}$, and $1 \mathrm{THz}$, respectively.

\section{Acknowledgement}

Authors are very thankful to Research Fellow Svetlana G. Bunchuk for her scrupulous attitude towards preparation of the samples, and Dr. Mikolay V. Sakhno for useful discussions.

This work was partly supported by Ukrainian Program of Basic Researches (grant No. 11/14-H) and joint project of Ukrainian National Academy of Sciences (grant No. 01-02-2012) and Siberian Branch of Russian Academy of Sciences (grant No. 15.1).

\section{References}

1. A.G. Davies, A.D. Burnett, W.H. Fan, E.H. Linfield and J.E. Cunningham, Terahertz spectroscopy of explosives and drugs // Materials Today, 11(3), p. 18-26 (2008). 
2. F. Sizov, A. Rogalski, THz detectors // Progr. Quant. Electr. 34(5), p. 278-347 (2010).

3. R. Appleby, H.B. Wallace, Standoff detection of weapons and contraband in the $100 \mathrm{GHz}$ to $1 \mathrm{THz}$ region // IEEE Trans. Antennas Propag. 55, p. 2944-2956, 2007.

4. Kai-Erik Peiponen, J. Axel Zeitler and Makoto Kuwata-Gonokami (eds.), Terahertz Spectroscopy and Imaging. Springer Series in Optical Sciences, 171, Heidelberg-New York-Dordrecht-London, 2013, p. 1-641.

5. Y.C. Choi, J. Shi, M. Pophristic, M.G. Spencer, and L.F. Eastman, C-doped semi-insulating GaN HFETs on sapphire substrates with a high breakdown voltage and low specific on-resistance // J. Vac. Sci. Technol. B, 25, p. 1836-1841 (2007).

6. M. Kuroda, H. Ishida, T. Ueda, and T. Tanaka, Nonpolar (11-20) plane AlGaN/GaN heterojunction field effect transistors on (1-102) plane sapphire // J. Appl. Phys. 102, Article ID 093703, 2007, p. 1-8.

7. M.S Shur and P. Maki (eds.), Advanced High Speed Devices, in: Selected Topics in Electronics and Systems, 51, World Scientific, Singapore, 2009, p. 1-187.

8. El Fatimy, S.B. Tombet, F. Teppe, W. Knap, D.B. Veksler, S. Rumyantsev, M. Shur, Terahertz detection by $\mathrm{GaN} / \mathrm{AlGaN}$ transistors // Electron. Lett. 42, p. 1342-1343 (2006).

9. L. Liu, B. Sensale-Rodriguez, Z. Zhang, T. Zimmermann, Y. Cao, D. Jena, P. Fay, and H. Xing, Development of microwave and terahertz detectors utilizing AIN/GaN high electron mobility transistors // Proc. 21st ISSTT, Oxford, 2010, p. 321-325.

10. P. Sangare, G. Ducournau, B. Grimbert et al., Experimental demonstration of direct terahertz detection at room-temperature in $\mathrm{AlGaN} / \mathrm{GaN}$ asymmetric nanostructures // J. Appl. Phys. 113, Article ID 034305, p. 1-6 (Jan. 2013).

11. Y. Tsividis, C. McAndrew, Operation and Modeling of the MOS Transistor, 3rd ed. Oxford University Press, New York, 2011, p. 1-726.
12. M. Sakhno, A. Golenkov, and F. Sizov, Uncooled detector challenges: Millimeter-wave and terahertz long channel field effect transistor and Schottky barrier diode detectors // J. Appl. Phys. 114, 164503 (2013).

13. W. Knap, M. Dyakonov, D. Coquillat et al., Field Effect Transistors for Terahertz Detection: Physics and First Imaging Applications // J. IRMMW-THz, 30, p. 1319-1337 (Dec. 2009).

14. R. Tauk, F. Teppe, S. Boubanga et al., Plasma wave detection of terahertz radiation by silicon field effects transistors: Responsivity and noise equivalent power // Appl. Phys. Lett. 89, Article ID 253511, p. 1-3 (Dec. 2006).

15. E. Ojefors, U.R. Pfeiffer, A. Lisauskas, H.G. Roskos, A $0.65 \mathrm{THz}$ focal-plane array in a quartermicron CMOS process technology // IEEE J. SolidState Circuits, 44, p. 1968-1976 (2009).

16. Antenna Engineering Handbook, 4th ed., J.L. Volakis (ed.). McGraw-Hill Companies, New York, 2007, p. 1-1755.

17. M. Sakhno, J. Gumenjuk-Sichevska and F. Sizov, Modeling of the substrate influence on multielement $\mathrm{THz}$ detector operation // J. IRMMW-THz, 35, p. 703-719 (2014).

18. F. Sizov, V. Petriakov, V. Zabudsky, D. Krasilnikov, M. Smoliy, and S. Dvoretski, Millimeterwave hybrid un-cooled narrow-gap hot-carrier and Schottky diodes direct detectors // Appl. Phys. Lett. 101, 082108 (2012).

19. C.A. Balanis, Antenna Theory: Analysis and Design. 3rd ed., Wiley-Interscience, A John Wiley \& Sons, Inc., 2005, p. 1-1072.

20. M. Sakowicz, M.B. Lifshits, O.A. Klimenko, F. Schuster, D. Coquillat, F. Teppe, and W. Knap, Terahertz responsivity of field effect transistors versus their static channel conductivity and loading effects // J. Appl. Phys. 110, Article ID 054512, p. 1-6 (Sep. 2011).

21. V.V. Popov, D.M. Yermolaev, K.V. Maremyanin et al., Detection of terahertz radiation by tightly concatenated InGaAs field-effect transistors integrated on a single chip // Appl. Phys. Lett. 104, Article ID 163508, p. 1-4 (2014). 